\title{
The effect of acute simvastatin administration on the ischaemia and reperfusion-induced ventricular arrhythmias in anaesthetized dogs
}

PhD Thesis

Gábor Kisvári

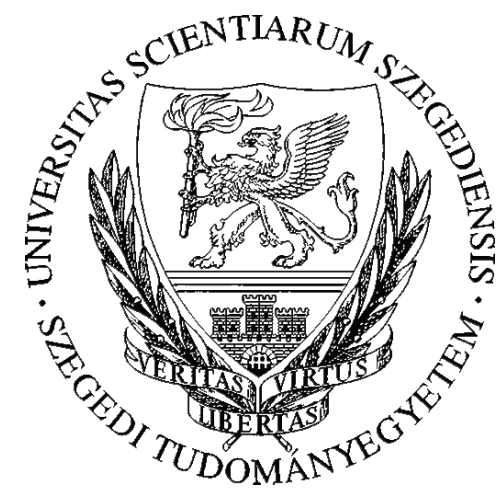

Supervisor: Prof. Dr. Ágnes Végh

Department of Pharmacology and Pharmacotherapy

Albert Szent-Györgyi Medical Center

University of Szeged, Hungary

2016 


\section{LIST OF PUBLICATIONS}

\section{Full papers}

1. Kisvári G, Kovács M, Gardi J, Seprényi G, Kaszaki J, Végh Á. The effect of acute simvastatin administration on the severity of arrhythmias resulting from ischaemia and reperfusion in the canine: Is there a role for nitric oxide? Eur J Pharmacol. 2014; 5;732:96-104. IF:2.13

2. Kisvári G, Kovács M, Seprényi G, Végh Á. The activation of PI 3-kinase/Akt pathway is involved in the acute effects of simvastatin against ischaemia and reperfusion-induced arrhythmias in anaesthetised dogs. Eur J Pharmacol. 2015; 15;769:185-94. IF:2.54 


\section{INTRODUCTION}

Severe ventricular tachyarrhythmias, resulting from coronary artery occlusion, are one of the main causes of sudden cardiac death (SCD). The prevention of the heart from these lifethreatening arrhythmias is one of the biggest challenges of modern cardiology. Despite advances in drug therapy, surgical and interventional cardiology, further research is required to explore new strategies that eventually may provide novel therapeutic interventions. One of these attempts was the study of Murry and his colleagues in 1986, which provided the first evidence that the heart is able to adapt to an ischaemic stress; i.e. short periods of ischaemia increase the tolerance of the heart to a subsequent more severe and prolonged period of ischaemia. This phenomenon was termed as 'ischaemic preconditioning'.

Another important step in the research of preconditioning was the recognition that certain drugs may induce protection similar to that of preconditioning. The idea of the "drug-induced preconditioning ,came from the assumption that if endogenous substances are, indeed, involved in the preconditioning-induced protection, and that their synthesis and the release become impaired under pathophysiological conditions, then drugs, which are able to replace or modify the production of these mediators, would elicit protection.

Recent clinical studies suggest that statins that were originally developed for the treatment of hypercholesterolemia may provide protection against the severe ventricular arrhythmias and sudden cardiac death. There is increasing experimental and clinical evidence that statins, beyond their lipid lowering effects, are able to reduce the incidence of fatal ventricular arrhythmias, which are the major cause of mortality in various cardiovascular diseases. Since cardioprotection by statins was also observed under normocholesterolemic conditions, it was proposed that these drugs might have cholesterol-independent, pleiotropic effects as well. These include plaque stabilization and improvement in vascular endothelial function, reduction in oxidative stressinduced injury, inhibition of inflammatory and thrombogenic responses. Most of these effects are thought to play a role in the anti-arrhythmic action of statins, and would explain the observed reduction in cardiac death in patients with statin treatment. The salutary effects, unrelated to the cholesterol lowering action of statins, are proposed to involve both nitric oxide (NO)-dependent and NO-independent mechanisms. For example, statins have been found to increase the generation of NO via eNOS activation, enhance the formation of prostanoids and induce hemeoxygenase- 1 . 
Perhaps, the most likely mechanism by which statins provide protection against ischaemia and reperfusion-induced arrhythmias is their ability to increase NO synthesis. There is substantial previous evidence that NO plays an essential role in both the early and delayed anti-arrhythmic effects of preconditioning, induced either by brief periods of coronary artery occlusion, cardiac pacing or heavy physical exercise. It was also demonstrated that these preconditioning stimuli, via the activation of eNOS, enhance NO production and modify myocardial function during ischaemia and reperfusion. More recently it was showed that pacing induces an immediate increase in eNOS activation and NO production, but it also causes an up-regulation of eNOS gene and protein expressions 12 and $24 \mathrm{~h}$ later.

Although the precise mechanism through which statins yield cardioprotection is still not well established, clinical and experimental studies suggest that NO, generated via the activation of eNOS, may play a mandatory role. The mechanisms by which statins may activate eNOS are even less understood; the prevention of the oxLDL-mediated down-regulation of eNOS mRNA and protein levels, as well as the up-regulation of eNOS by blocking the synthesis of those isoprenoid intermediates of the cholesterol synthesis pathway that are involved in the posttranslational modification of proteins which regulate eNOS expression, were suggested as potential mechanisms for eNOS activation during chronic statin administration. There are, however, only a few studies which have attempted to examine the acute effects of statins; these were related to the assessment of infarct size and post-ischaemic contractile dysfunction. One of the possible pathways that have been proposed to activate eNOS through phosphorylation within minutes would be the PI 3-kinase/Akt signalization. Indeed, Kureishi and his colleagues (2000) have demonstrated that following acute simvastatin administration, the activation of the PI 3kinase/Akt/eNOS cascade is involved in the increase of NO synthesis in endothelial cells. Similarly, Wolfrum and his colleagues (2004) showed that in anaesthetized rats subjected to coronary artery occlusion, the administration of simvastatin just prior to reperfusion, markedly reduced infarct size, increased PI 3-kinase activity, as well as Akt Ser473 and eNOS Ser1177 phosphorylation. There is, however, no study which has examined how a single dose of simvastatin influences the acute ischaemia and reperfusion induced severe ventricular arrhythmias. To answer this question, two studies were designed and performed. 


\section{AIMS OF THE STUDY}

The purpose of the present thesis was to answer the question whether a single dose of simvastatin would modify the acute ischaemia and reperfusion induced severe, often lifethreatening ventricular arrhythmias. If so, what might be the mechanism? We have designed two separate studies in order to obtain answers for these questions, and the results were published in the enclosed publications. Our questions were as follows:

a) There is no sufficient information as to whether the acute simvastatin administration would elicit a similar salutary effect. Therefore, we designed studies in which we have examined in our established canine model, whether a single bolus injection of simvastatin prior to an ischaemia and reperfusion insult would modify the severity of ventricular arrhythmias. We have also examined whether nitric oxide (NO) is involved in the acute effect of simvastatin.

b) Since we have found that the acute administration of simvastatin provides marked protection against the ischaemia and reperfusion-induced severe ventricular arrhythmias and that this antiarrhythmic effect involves the generation of NO by simvastatin, in a separate study we have now examined whether this NO formation is mediated through the rapid activation eNOS via the stimulation of the PI 3-kinase/Akt signalization. For this purpose we used the PI-3kinase inhibitor wortmannin prior to the administration of simvastatin.

\section{MATERIALS AND METHODS}

\section{Surgical preparation}

The animals were anaesthetized with pentobarbital $\left(30 \mathrm{mg} \mathrm{kg}^{-1}\right.$ intravenous sodium pentobarbitone). The right femoral artery was prepared and catheterized, through which the dogs

were further anaesthetized with a mixture of chloralose and urethane (60 and $200 \mathrm{mg} \mathrm{kg}^{-1}$, respectively) to maintain anaesthesia.

Polyethylene catheters were inserted into the right femoral artery for monitoring arterial blood pressures, and via the left carotid artery into the left ventricle (LV) for the measurement of systolic (LVSP) and end-diastolic (LVEDP) pressures.

The chest was opened at the fifth intercostal space and the anterior descending branch of the left coronary artery (LAD) was prepared for occlusion just proximal to the first main diagonal branch. Distal to the occlusion site a smaller side branch of the same artery was also prepared and cannulated for the local administration of drugs (simvastatin, L-NAME, wortmannin and DMSO) 
and vehicle. Another catheter was positioned into the coronary sinus to obtain blood samples for the assessment of plasma nitrate/nitrite (NOx) levels. In some dogs from each group, the left circumflex (LCX) coronary artery was also prepared to measure coronary blood flow.

\section{Measurement of ischaemia severity}

The severity of myocardial ischaemia was evaluated by changes in the epicardial STsegment and in the degree of inhomogeneity of electrical activation. These were measured by a composite electrode (containing also four unipolar electrodes by which changes in ST-segment $[\mathrm{mV}]$ were detected) positioned within the potentially ischaemic area. The greatest delay in activation within the ischaemic area following coronary artery occlusion was expressed in ms.

\section{Assessment of ventricular arrhythmias}

Ventricular arrhythmias were assessed according to the Lambeth conventions. The total number of ventricular premature beats (VPBs), the incidence and the number of episodes of ventricular tachycardia (VT), and the incidence of ventricular fibrillation (VF) were assessed during the occlusion period. During reperfusion, only the incidence of VF, which is a fatal event in this species, was determined. Dogs that were alive 1-2 min after reperfusion were considered to be survivors.

\section{Measurement of plasma nitrate/nitrite (NOx) levels}

These were performed as described previously. Plasma nitrate/nitrite $\left(\mathrm{NO}_{\mathrm{x}}\right)$ concentrations were determined by means of the Griess reaction in blood samples taken from the coronary sinus at various time intervals. After preparation of blood samples, the absorbance of the azo compound was measured spectrophotometrically at a wavelength of $540 \mathrm{~nm}$ and the total nitrate/nitrite $\left(\mathrm{NO}_{\mathrm{x}}\right)$ concentration $\left(\mu \mathrm{mol} \mathrm{l}^{-1}\right)$ was determined using a standard calibration curve of $\mathrm{NaNO}_{2}$ and $\mathrm{NaNO}_{3}$.

\section{Determination of eNOS phosphorylation by Western blot}

Freshly excised tissue samples from the ischaemic and non-ischaemic regions of the left ventricular myocardial wall were immediately frozen in liquid nitrogen and stored at $-80^{\circ} \mathrm{C}$. $100 \mu \mathrm{g}$ of protein extracts were resolved using 8\% sodium dodecyl sulphate-polyacrylamide gel electrophoresis and blotted on polyvinylidene fluoride membranes. The blots were immunolabeled overnight with a monoclonal mouse anti-eNOS primary antibody (pS1177) diluted to 1:2500, followed by $1 \mathrm{~h}$ incubation with an HRP-conjugated anti-mouse rabbit 
secondary antibody in a dilution of 1:8000. Band densities were detected with the ECL Plus kit and developed on Amersham Hyperfilm ${ }^{\mathrm{TM}}$. Pixel intensities of each band were measured using ImageJ software (NIH). Three parallel Western blots were performed for the statistical analysis using Bonferroni correction. On the blots each examined group was compared to the shamcontrols. For verifying equal loading, PVDF membranes were labelled with Coomassie-Blue.

An integrated optical density value (the sum of each pixel value corrected to the background) was formed by drawing equal size boxes around the bands. The intensities obtained from both the total and peNOS bands were normalized to this integrated value.

\section{Determination of the functional activity of eNOS by radio immunoassay}

This was performed using a NOS activity assay kit based on the biochemical conversion of $\left[{ }^{3} \mathrm{H}\right]$ L-arginine to $\left[{ }^{3} \mathrm{H}\right]$ L-citrulline by NOS. From the tissue samples (100 mg) membrane proteins were isolated, homogenized in ice-cold homogenization buffer, and centrifuged at 2000 $\mathrm{g}$ for $15 \mathrm{~min}$. The supernatant was then ultra-centrifuged at $50000 \mathrm{~g}$ for $45 \mathrm{~min}$ and the pellet (membrane fraction) was re-suspended in the homogenization buffer. A liquid scintillation counter was used to determine eNOS activity by measuring the amount of the radio-labelled citrulline formed during the reaction, and expressed as the percentage of the total counts corrected with the background counts per minute.

\section{Assessment of superoxide production}

This was determined by dihydroethidium (DHE) fluorescence staining. Tissue blocks, excised from the ischaemic myocardial wall were embedded in optimal cutting temperature compounds. Cryosections $(20 \mu \mathrm{m})$ were produced, stained with DHE $\left(1 \mu \mathrm{mol} 1^{-1}\right.$, dissolved in $\mathrm{pH}$ 7.4 phosphate buffer solution), and incubated at $37^{\circ} \mathrm{C}$ for $30 \mathrm{~min}$ in a dark humidified chamber. A negative control was obtained by blocking the reaction with N-acetyl-L-cysteine (NAC, 100 mmoll $^{-1}$.) Both from the stained and the negative control samples 10 to 15 serial images were captured by a confocal laser scanning microscope. The intensity of the fluorescent signals were analyzed by ImageJ software (NIH) and expressed in arbitrary units.

\section{Preparation of simvastatin solution}

Before the application of simvastatin it has to be converted into an active form. A stock solution containing $25 \mathrm{mg}$ simvastatin, dissolved in $625 \mu \mathrm{l}$ ethanol and $937.5 \mu 10.1 \mathrm{~N} \mathrm{NaOH}$, was prepared and incubated at $50^{\circ} \mathrm{C}$ for 2 hours. Then the $\mathrm{pH}$ of the solution was adjusted to 7.0 
with $1 \mathrm{~N} \mathrm{HCl}$ and stored at $-20^{\circ} \mathrm{C}$ until use. Immediately prior to the experiments an aliquot was taken and diluted in distilled water to obtain the appropriate dose.

\section{Experimental protocols}

\section{Experimental protocol for the assessment of acute simvastatin administration on the ischaemia and reperfusion-induced arrhythmias}

Dogs were randomly selected to form four experimental groups. Control dogs were administered the solvent of simvastatin $\left(0.5 \mathrm{ml} \mathrm{min}^{-1}\right)$ in intracoronary injection (over $\left.5 \mathrm{~min}\right)$ and subjected to a 25 min occlusion and then reperfusion of the descending branch of the left coronary artery (LAD). Fifteen dogs were received activated simvastatin in a dose of $0.1 \mathrm{mg} \mathrm{kg}^{-1}$ by the same route, 5 min prior to the onset of the occlusion. In another two groups, L-NAME was given in a dose of $5 \mathrm{mg} \mathrm{kg}^{-1}$ also in slow intracoronary injection, $15 \mathrm{~min}$ before the solvent and the simvastatin administration. Eight dogs were used as sham-operated controls. These dogs were only instrumented, without subjecting them to any treatment, and from which, after the euthanasia, tissue samples were harvested for measuring eNOS phosphorylation and superoxide production. At the end of the experiments the hearts were stopped by an excess of the anaesthetic, and myocardial tissue samples were collected from both the ischaemic and the non-ischaemic regions of the left ventricular wall for further analyses. In at least 5 dogs of each group sample taking was performed at the end of the 25 min occlusion period, whereas in dogs that had been subjected to reperfusion, tissue samples were collected either $5 \mathrm{~min}$ after reperfusion (these animals were considered as survivors) or at the time when the fibrillation was observed.

\section{Experimental protocol for the assessment the role of PI 3-kinase/Akt pathway in protection provided by the acute simvastatin administration}

In the second series of the experiment, the dogs were randomly selected into five experimental groups. The control and the simvastatin groups were similar to the groups mentioned above. In two other groups, wortmannin alone or together with simvastatin was given in a dose of $1.5 \mathrm{mgkg}^{-1}$ in slow intracoronary injection, 15 min before the administration of the solvent and simvastatin. Additional $13 \mathrm{dogs}$ were received $0.1 \%$ dimethylsulphoxide (DMSO), the solvent of wortmannin. Data obtained from 8 dogs in the control and from 6 dogs in the simvastatin groups were already used in the first series of experiments. 


\section{RESULTS}

I/1. Local intracoronary injection of simvastatin and the solvent of simvastatin did not substantially modify the haemodynamic parameters and blood flow, measured on the LCX coronary artery. In contrast, the administration of L-NAME significantly elevated arterial blood pressure and reduced heart rate without substantially modifying the other haemodynamic parameters. These haemodynamic changes in the L-NAME treated dogs were still present before the onset of the LAD occlusion. Occlusion of the LAD resulted in significant reductions in haemodynamic parameters.

I/2. In control dogs, occlusion of the LAD resulted in a high number of VPBs and episodes of VT. Furthermore, $56 \%$ of the dogs fibrillated during occlusion and no control dog survived reperfusion. Local administration of simvastatin significantly reduced these arrhythmias during occlusion, and increased survival compared to the controls. L-NAME did not substantially modify arrhythmia severity during occlusion and reperfusion, but it significantly attenuated the antiarrhythmic effect of simvastatin. Interestingly, L-NAME, however, did not affect the protective effect of simvastatin against the occlusion-induced ventricular fibrillation.

I/3. In control dogs, occlusion of the LAD resulted in immediate and significant increases both in the epicardial ST-segment and the degree of inhomogeneity of electrical activation. These changes were significantly less pronounced following simvastatin administration. Although LNAME itself did not modify these indices of ischaemia severity, it abolished the anti-ischaemic effects of simvastatin.

I/4. When the LAD was occluded in control animals, a transient elevation occurred in the plasma NOx levels (around 7 min of the ischaemia), after which NOx started to decrease and became significantly lower than the initial baseline values. In contrast, the administration of simvastatin elevated NOx levels almost over the entire occlusion period. This effect was completely abolished by the prior administration of L-NAME. Reperfusion of the ischaemic myocardium evoked similar increases in NOx levels in all groups.

I/5. The activity of eNOS was assessed by measuring eNOS phosphorylation with Western blot, whereas the functional activity of the enzyme was determined using radio immunoassay. Occlusion of the LAD significantly reduced the activity of eNOS by the end of the occlusion period. Simvastatin preserved or even increased both the phosphorylation and the functional 
activity of the enzyme. Although L-NAME alone did not modify the ischaemia-induced reduction in eNOS activity, it abolished the simvastatin-induced activation, but not the phosphorylation of the enzyme. After reperfusion, the function of eNOS was rapidly regained in the control dogs, maintained in the simvastatin treated dogs, and remained inhibited in dogs given L-NAME.

I/6. Compared with the sham controls, in dogs subjected to ischaemia and reperfusion a marked increase in superoxide production occurred soon after the reopening of the coronary artery. This ischaemia and reperfusion-induced generation of superoxide was significantly suppressed by the administration of simvastatin; an effect which was reversed by L-NAME.

I/7. This was measured in order to assess the potential direct electrophysiological effects of simvastatin. In control dogs a marked increase developed in QTc interval within 5 min of the occlusion and this was maintained during the whole ischaemic period. Such a prolongation of the QTc interval was abrogated with simvastatin no matter whether L-NAME was present or not.

II/1. There were no considerable changes in the haemodynamic parameters following the local intracoronary injection of simvastatin and wortmannin, as well as of the vehicle of these drugs. Although occlusion of the LAD resulted in significant reductions in haemodynamic parameters in all groups, these changes were not substantially different among the groups. The compensatory blood flow changes, occurring during the occlusion of the LAD on the LCX coronary artery, were not significantly modified by any drug treatment.

II/2. The administration of wortmannin in the simvastatin treated dogs significantly reduced the antiarrhythmic protection. Thus in the presence of wortmannin the number of VPBs, the incidence and number of episodes of VT were again increased, and as with the controls (C and DMSO groups), no dog in the $\mathrm{W}+\mathrm{S}$ group survived reperfusion. However, wortmannin, like LNAME before did not modify the effect of simvastatin on the ischaemia-induced VF. Compared to the controls, neither wortmannin, nor DMSO, the vehicle of wortmannin, affected the severity of arrhythmias resulted from a 25 min LAD occlusion and reperfusion. In these groups none of the dogs survived reperfusion.

II/3. While simvastatin markedly suppressed the indices of ischaemia severity, its effect was completely abolished in the presence of wortmannin. Inhibition of the PI 3-kinase by wortmannin had no significant effect on these ischaemia-induced inhomogeneity and ST-segment changes.

II/4. The effect of simvastatin on eNOS activity was abolished in the presence of wortmannin, although wortmannin alone did not affect the ischaemia-induced changes in NOS 
phosphorylation. When the coronary artery was re-opened, it can be seen that NOS regained very rapidly. Thus following reperfusion the phosphorylation of eNOS was increased in almost all groups, except those that had been received wortmannin prior to the 25 min occlusion and reperfusion of the LAD.

II/5. The administration of wortmannin in control dogs abolished the early increase of NO metabolites, and it completely abrogated the NO preserving effect of simvastatin over the entire occlusion period. DMSO had no effect on NO bioavailability during occlusion and reperfusion. II/6. The administration of DMSO, as well as of wortmannin itself, did not affect the ischaemia/reperfusion-induced generation of superoxide, but wortmannin reversed the effect of simvastatin on superoxide production.

II/7. Similar to L-NAME, in the presence of wortmannin the effect of simvastatin on the QTc interval was still maintained.

\section{DISCUSSION}

\section{New findings}

1. We have provided evidence that a single dose of simvastatin, applying just prior to a 25 min period of coronary artery occlusion in anaesthetized dogs, results in profound protection against those severe ventricular arrhythmias that are, in most instances, responsible for sudden cardiac death. We have also pointed out that this antiarrhythmic effect of simvastatin, at least in part, is due to an increased nitric oxide production, resulting from the rapid activation of the endothelial nitric oxide synthase (eNOS) by simvastatin, since the protective effect of simvastatin was almost completely abolished in the presence of the NOS enzyme inhibitor L-NAME.

2. We have also shown that the simvastatin-induced rapid eNOS activation results from the stimulation of the IP3/Akt pathway, since the inhibition of the IP3-kinase with wortmannin, attenuates eNOS activation and, consequently, abolishes the antiarrhythmic protection.

\section{The effect of acute simvastatin administration on ischaemia and reperfusion ventricular arrhythmias. The role of nitric oxide.}

There are only few studies that raised up the question, whether statins, following acute administration, would influence the consequences of ischaemia and reperfusion, including the life threatening ventricular arrhythmias. Furthermore, since many of the beneficial effects of statins 
have been shown to involve increased synthesis of nitric oxide, and since we have a number of previous evidence that NO plays an essential role in the protection against the ischaemia-induced early arrhythmias, we have now examined whether the effect of simvastatin on arrhythmias is also due to the activation of eNOS and the subsequent increase in NO formation.

The results show that in anaesthetized dogs the administration of a single dose of simvastatin markedly reduced the number of VPBs and episodes of VT, the incidence of VT and VF that resulted from a 25 min occlusion of the LAD and increased survival from the combined ischaemia and reperfusion insult, compared with the untreated controls. Furthermore, simvastatin significantly reduced the severity of ischaemic changes during coronary artery occlusion. These salutary effects of simvastatin administration were associated with increased eNOS activity since the phosphorylation of eNOS (determined by Western blot) and the functional activity of this enzyme (determined by RIA) were significantly higher during ischaemia and reperfusion in the simvastatin treated dogs than in the solvent treated controls. This activation of eNOS by simvastatin resulted in an increased generation of NO during occlusion and also a decrease in superoxide production following reperfusion. As we have shown previously, the preservation of NO bioavailability during coronary artery occlusion by preconditioning or by donating NO, attenuates the reperfusion-induced marked increases of superoxide production, since NO may regulate the generation of superoxide during reperfusion. These effects are certainly can be associated with the antiarrhythmic effect of simvastatin. Furthermore, the fact that L-NAME prevented the simvastatin activated enzyme to form NO (inhibits the L-arginine-NO pathway without affecting enzyme phosphorylation) and attenuated or even abolished most of the salutary effects of simvastatin, supports the hypothesis that the eNOS activated NO formation plays a crucial role in the protective effect of simvastatin against arrhythmias.

We have raised the question, how the activation of eNOS takes place following a single dose of simvastatin. There are a few studies in the literature, which show that statins can rapidly activate eNOS by stimulating the phoshatidylinositide 3-kinase/Akt (PI 3-kinase/Akt) pathway. In the second part of the experiments we examined, whether the antiarrhythmic effect of acute simvastatin administration involves the rapid activation of the PI 3-kinase/Akt/eNOS signalling pathway. For this purpose we have also used wortmannin, a potent and specific inhibitor of PI 3kinase, and administered in dogs prior to the bolus injection of simvastatin. We have found that in the presence of wortmannin most of the salutary effects of simvastatin were attenuated, or even 
abolished. Thus the number of VPBs, the number of episodes of VT and the incidence of VT during occlusion were again increased, and compared with the simvastatin treated group, in which $40 \%$ of the dogs survived reperfusion, in the wortmannin + simvastatin group no animal survived the combined ischaemia and reperfusion insult. The pre-treatment with wortmannin significantly attenuated the simvastatin induced activation of eNOS and the subsequent NO production, as well as the decreases in superoxide generation. The marked anti-ischaemic effect of simvastatin was also abolished by the administration of wortmannin.

There is still ongoing debate as to whether the reduction in tachyarrhythmias (VT and VF) that are responsible in most instances for sudden cardiac death, is due to the anti-ischaemic effect of statins, or there might be a more direct electrophysiological action of these drugs, which would explain their marked antiarrhythmic effect. Although we have found that simvastatin results in pronounced anti-ischaemic effect, we suppose that simvastatin might possess a direct antiarrhythmic effect as well. The evidence for this assumption may come from two observations; i.e. (i) despite the abolition of the anti-ischaemic effect of simvastatin by L-NAME and wortmannin, the protective effect of simvastatin against the ischaemia-induced ventricular fibrillation was still present, and (ii) neither L-NAME nor wortmannin did not influence the reduction in QTc interval, resulted from simvastatin administration. However, interestingly, both inhibitors abolished the protective effect of simvastatin against the reperfusion-induced VF. The explanations for this dichotomy could be many and varied, including differences in the underlying mechanisms of the various arrhythmia types induced by the acute ischaemia, as well as differences in the local and systemic regulatory influences of NO on arrhythmia mechanisms. These latter may involve, for example, the modulation of the effect of autonomic tone on the myocardium and of gap junction function, as well as the regulation of free radical formation by NO.

Nevertheless, the fact that L-NAME and wortmannin did not influence the protective effect of simvastatin against the occlusion-induced ventricular fibrillation suggests that in the anti-fibrillatory effect of simvastatin, there might be an NO-independent and, perhaps, a more direct electrophysiological mechanism. This assumption is supported by the results of the QTc interval measurements. These show that simvastatin almost completely inhibited the ischaemiainduced prolongation of the QTc interval, and this effect was not modified by the administration of L-NAME or wortmannin. Recent electrophysiological studies suggest that statins influence 
impulse conduction, improve cardiac repolarization and suppress cardiac excitability perhaps by directly and selectively affecting ion channels in cardiomyocytes (e.g. Kv4.3). Certainly, longterm statin treatment by modulating the lipid portions of the sarcolemma, which contain the ion channel regulatory proteins and signalling molecules (lipid rafts), influence the ion channel conduction and ion transport, but it is not known whether such a mechanism would also account for the acute administration of statins. Nevertheless, considering that polyunsaturated fatty acids, which alter the structure of the sarcolemmal phospholipids, were able to evoke immediate antiarrhythmic effect, we may speculate that statins perhaps also possess such acute modulator properties on the lipid portions of the membrane. This, by causing favourable changes in ion transport or by stabilizing the membrane, would lead to arrhythmia suppression during ischaemia.

In contrast, the fact that L-NAME and wortmannin abrogated the protective effect of simvastatin against the reperfusion-induced VF suggests that simvastatin through an NOdependent way reduces superoxide production and hence the occurrence of VF during reperfusion. Consequently, this protection disappears by inhibiting the formation of NO.

Summarizing our results, we have shown that a single bolus injection of simvastatin markedly reduces the ischaemic changes and the incidence and severity of ventricular arrhythmias that results from a $25 \mathrm{~min}$ occlusion and reperfusion in anaesthetized dogs. Furthermore, we have demonstrated that the simvastatin-induced marked antiarrhythmic effect largely depends on the generation of NO that results from the rapid activation of eNOS via the stimulation of the PI 3-kinase/Akt pathway. Thus we think that the activation of PI 3kinase/Akt/eNOS cascade and the subsequent increased formation of NO play a crucial role in the antiarrhythmic effect of acute simvastatin administration, but a direct and presumably NOindependent mechanism cannot be ruled out.

We are convinced that NO bioavailability during occlusion plays an essential role in arrhythmia generation both during occlusion and reperfusion. Thus any manoeuvre, which increases or, at least, maintains NO formation during occlusion may protect the myocardium against arrhythmias, most probably through the regulation of those local and systemic mechanisms by NO that are implicated in arrhythmia generation, such as the autonomic tone, gap junctional function, or the formation of free radicals, etc. The latter is particularly important in the generation of the reperfusion-induced severe ventricular arrhythmias, and NO by reducing free radical formation may suppress the occurrence of arrhythmias during reperfusion. Since both 
L-NAME and wortmannin abolished the suppressing effect of simvastatin on the reperfusioninduced superoxide production and subsequently increased the incidence of VF during reperfusion, it seems more than likely that this effect of simvastatin is NO-dependent and it attains via the activation of the PI 3-kinase/Akt/eNOS pathway. On the other hand, those of our findings which show that the inhibition of NO formation does not influence the protective effect of simvastatin against the occlusion-induced VF indicates an NO-independent, direct electrophysiological mechanism in the anti-fibrillatory effect of simvastatin.

\section{ACKNOWLEDGEMENT}

First and foremost, I would like to express my gratitude to my principal supervisor, Prof. Dr. Ágnes Végh, who helped me acquire the basic experimental skills, and I am especially grateful for her patience and daily assistance in my performing the studies, preparing for conferences, writing publications and my thesis. I am also grateful to Prof. Dr. András Varró for providing me with the opportunity to carry out my scientific work and for initiating my scientific career in the Department of Pharmacology and Pharmacotherapy under his valuable scientific guidance.

The members of the department, especially Dr. Márton Gönczi, Gottfried Miskolczi, Dr. László Juhász, Dr. Attila Kiss and Dr. Mária Kovács have contributed immensely to my personal and professional activities. The team has been a source of friendships, good advice and collaboration. Special thanks go to Erika Bakó and Irén Biczókné. Apart from their precious professional expertise in conducting animal experiments they were both worthy friends and made working hours more colorful. I would like to give my sincere thanks to Dr. György Seprényi, who created the possibilities for doing the fluorescent microscopy experiments in the Institute of Medical Biology.

This work would not have been possible without the help and support of my researcher colleagues in Szeged. I have been able to learn and develop considerably through this and I would never have achieved my goals without them.

I wish to thank my close and distant friends for supporting me during the years, helping me to get through challenging times. Lastly, I am very grateful to and wish to thank my family. 